\title{
LA FUGA DE CEREBROS EN ESPAÑA, ¿HACIA DÓNDE DEBEN DIRIGIRSE LAS POLÍTICAS PÚBLICAS PARA FRENAR EL ÉXODO?
}

\author{
The brain drain in spain, where should be directed to public policy to stop the \\ exodus?
}

Eva García Redondo

Fecha recepción: 20/07/2016

Fecha aceptación: 12/11/2016

RESUMEN: Los movimientos migratorios, por razones laborales o formativas, han sido, históricamente, objeto de reconocido análisis desde la Economía. En los últimos cincuenta años, la preocupación ha transcendido ese ámbito científico convirtiéndose, si bien con menos fuerza y expansión, en tópico trabajado desde la Pedagogía. La internacionalización de la educación, de sus instituciones y títulos, ha contribuido a un proceso migratorio que, pudiendo ser positivo, se ha tornado en una puerta abierta al abandono de talentos de aquellos países con menos posibilidades de absorción de mentes. Distinguimos, por tanto, entre intercambio -como factor recomendable, de crecimiento, aprendizaje y retorno- y fuga o abandono -como resultado de un proceso de movilidad unidireccional no retornable-. El estudio del documento "The Global Talent Competitiveness Index 2015-2016 (GTCI). Talent Attraction and International Mobility", publicado hace tan solo unos meses, nos permite cuestionamos por qué España se perfila como uno de los países que más "favorece" la salida nacional de cerebros comparando esta situación con la de otros países de la Unión. Para ello, recurrimos al análisis de las estrategias y políticas de retención de cerebros que están promoviendo los Estados más preocupados con el abandono de talentos.

PALABRAS CLAVE: Fuga de cerebros, España, internacionalización, talento, educación.

ABSTRACT: Migratory movements, for work and / or training reasons, have been historically recognized object of analysis from Economics. In the last fifty years, the concern has transcended the scientific field becoming a topic worked within Pedagogy. The internationalization of education, its institutions and titles, has contributed to a migration process that can be positive, it has turned into an open abandonment of talent from countries with less possibilities absorbing minds. Therefore, we distinguish among exchange recommendable factor to grow, learn and return- and human capital flight, as result of a process of unidirectional mobility nonrefundable. The study of "The Global Talent Competitiveness Index 2015-2016 (GTCI). Talent Attraction and International Mobility", published only a few months ago, we ask ourselves why Spain is emerging as one of Europe's members most "favored" by brain drain, comparing this situation with other countries in the Union. Herefrom, we turn to the study of strategies and retention policies brains are promoting the most concerned States with the brain drain.

KEYWORDS: Brain drain, Spain, internationzalization, talent, education. 


\section{Introducción}

La Sociedad Global o Sociedad del Conocimiento en la que nos encontramos propone una serie de problemáticas sociales que confirman la necesidad de avanzar hacia ciertas políticas de mejora de la calidad educativa y laboral. Estas últimas se centran en el estudio de los egresados (esencialmente universitarios), considerando que el análisis de los procesos de producción del conocimiento pueden y, además, deben ser mejorados para garantizar una integración profesional con ciertas garantías de éxito. Consideramos esencial el esfuerzo que los países del entorno europeo han llevado a cabo a través de la incorporación en sus agendas nacionales de vías políticas, sociales y económicas que permiten el intercambio de profesionales (Alcón, 2011). Gracias a ellas, hoy en día nadie duda de la importancia que tiene en la cualificación de expertos el poder disfrutar de tiempos y espacios de formación en el puesto de trabajo, máxime cuando estos se refieren a contextos de excelencia alejados de los países de origen. Se entiende, por tanto, que las características, idiosincráticas y originales, de una nación que no es la de nacimiento refieren posibilidades a ese trabajador del siglo XXI que busca en el exterior oportunidades, insistimos, no solo de cualificación profesional, sino de evolución y crecimiento personal y social. Esto es lo que Docquier y Rapoport (2012) defienden cuando afirman que "the recent empirical literature shows that high-skill emigration need not deplete a country's human capital stock and can generate positive network externalities" (p.681).

Atraer el talento internacional o lo que es lo mismo, albergar a los mejores académicos e investigadores del mundo es la finalidad por la que todos los países implicados en la "Estrategia Europa 2020" llevan trabajando ya algunos años. En este sentido, la excelencia científica debería ser el criterio básico y prioritario por el que se rigiera la migración cualificada, sin embargo, la crisis social, política y económica que estamos viviendo en la última década en Europa fomenta que esta se explique en base a otros factores. Como bien se encarga de plantear Sennett (2000), "quedarse quieto equivale a quedar fuera de juego" (p.91) pero optar por una movilidad de no retorno, es decir, por una pérdida nacional de capital humano supone incrementar la desigualdad entre territorios. Entendemos, por tanto, que la virtud está en ofrecer posibilidades de acción que no beneficien a unos países a la vez que perjudiquen a otros o lo que es lo mismo, que los flujos de migración altamente cualificada no sean siempre unidireccionales. Como afirman Valero, Mediavilla, Valero y Coca (2015), "las nuevas tendencias de movilidad humana han transformado el mapa migratorio mundial y la globalización ha participado de manera importante en el desplazamiento, asentamiento y metamorfosis de los perfiles, causas y consecuencias de los movimientos migratorios con respecto a hace cien años" (p. 43). De este modo, comprobamos cómo la internacionalización, a la que se suele aludir como realidad europea, no es más que el resultado de la prioritaria innovación tecnológica y de las propias acciones productivas (Sassen, 2007; Castells, 1996). Entran aquí en juego los conocidos como "push/pull factors" cuya presencia contribuye, en sendos casos, a dirigir la migración de naciones más limitadas a aquellas con mayores posibilidades de desarrollo. Lejos queda la idea clásica de que la movilidad afecta, exclusivamente, a los países pobres, puesto que, hoy en día, la fuga de talentos responde a un conjunto de factores mucho más amplio que, en cualquier caso, diagnostica una precariedad del mercado laboral (Alcañíz y Querol, 2015). El paro, las posibilidades de progreso y carrera profesional, las políticas de atracción de trabajadores, la cultura y el idioma, las tendencias político-sociales reinantes, incluso el grado y área de cualificación son solo algunas de las causas que nos hacen distinguir entre una internacionalización voluntaria y una obligada siendo esta última la que, para Santos (2013), se convierte en "un buen analizador de la cuestión juvenil pues supone una mutación ulterior de la precariedad que los jóvenes ya 
vivían y que ahora se convierte en una nueva dimensión de la precariedad: la de la migración cualificada ampliada" (p. 125).

Esta problemática, de amplia trayectoria en el campo de la Economía, es relativamente reciente en el de la Pedagogía. Por tanto, son todavía escasos los trabajos que aluden a esta desde una perspectiva educativa si bien, en los últimos 50 años (Puyol, 2014), esta tendencia se está viendo aminorada. Partiendo de los planteamientos que nos remite la literatura especializada, nuestro interés investigador se concentra, por un lado, en descubrir cuál es la situación actual que promueve ese tipo de movilidad tan concreta y, por otro, en conocer por qué España es un país paradigmático en lo que se refiere a la salida nacional de trabajadores cualificados en comparación con otros países de la Unión. Utilizaremos, para ello, la metodología de revisión bibliográfica al considerar que esta es la más adecuada para alcanzar los objetivos previstos, mostrando especial interés en el estudio del informe internacional "The Global Talent Competitiveness Index 2015-2016 (GTCI). Talent Attraction and International Mobility" (Lanvin y Evans, 2015).

\section{Orígenes históricos de la migración altamente cualificada.}

En los años 60 se comienza a observar en el mundo flujos migratorios que poco tienen que ver con los reconocidos hasta el momento (Brandi, 2006; Luchilo, 2015). Las migraciones a partir de esta década sugieren un cambio en la forma de concebir la movilidad, esencialmente porque quienes provocan este hecho ya no solo son hombres y mujeres con limitaciones competenciales, sino que, progresivamente, se van uniendo a la misma personas con cualificaciones medias y altas, es decir, profesionales a quienes las características socioeconómicas de su país les ofrece una oportunidad fuera de los mismos para realizarse a nivel profesional y, por ende, personal. Se acuña así el término "fuga de cerebros" para describir una situación, en el momento novedosa, como es la emigración de científicos y tecnólogos de Europa a América del Norte y la afluencia al Reino Unido de ingenieros y científicos de la India.

No es casual que, en este mismo momento, Schultz enuncie la Teoría del Capital Humano (1961). Su tesis principal reconoce que la educación es una inversión, es decir, una forma de capital que describe, posiciona y justifica el devenir de cada nación y que, por tanto, su apuesta es una ventaja de futuro. En este sentido, comprobamos cómo la propuesta de circulación de cerebros ("brain circulation"), justa y fructífera como se encarga de defender Reyes (2015), es tan solo una idea romántica que no encuentra espacio en la tendencia denominada "brain drain". La fuga de talentos se convierte, así, en una realidad que se aleja, progresivamente, del supuesto intercambio al tratarse de "un éxodo que suponía la pérdida de las personas mejor preparadas de los países de origen y el beneficio de su cualificación por parte de los territorios de destino que, además, no tenían que financiar la educación de esas personas" (Puyol, 2014, p.216). Durante cerca de 30 años, esta tendencia es mantenida favoreciendo a las naciones receptoras, no ofreciendo criterios de igualdad para el crecimiento y la competitividad.

En los años 90, un nuevo paradigma consigue hacerse un hueco en el discurso científico. El "brain gain" rompe con la legitimación, poder y estabilidad de los países más desarrollados proponiéndoles como medio, y ya no como fin, para el avance de los territorios inicialmente menos favorecidos. La opción ya no es la fuga sin retorno sino el paso, más o menos extenso, por una sociedad extranjera entendido como oportunidad para preparar la vuelta de un capital humano más preparado y competitivo a la de origen, lo que, en palabras de Sennett (2000), es la búsqueda de ese "subidón estimulante" preciso para el crecimiento social y personal de todo individuo. Bajo lo descrito por este paradigma, todas las 
comunidades implicadas salen beneficiadas: por un lado las receptoras porque aprovechan las potencialidades de unos trabajadores formados a coste cero $\mathrm{y}$, por otro, las de origen porque reciben profesionales cualificados en los más altos niveles de investigación y desarrollo, capaces de transferir el conocimiento adquirido a su realidad concreta, es decir, al marco de acción. Este nuevo enfoque permite ampliar el conjunto de beneficiarios produciéndose un auténtico proceso de internacionalización (González, Coca, Valero y Aguilar, 2015). Los flujos de intercambio suponen, tal y como venimos aludiendo, una nueva manera de entender la migración altamente cualificada que afecta, incluso, a los países tradicionalmente ricos. Sin embargo, la crisis a la que antes nos referíamos ha supuesto un freno a esta tendencia. En la última década, la fuga de cerebros se perfila, nuevamente, como una de las principales desventajas con las que ha de lidiar la Sociedad Global. El desempleo, la supracualificación, la precariedad laboral y la paralización del mercado productivo han hecho que países como España se hayan visto enfermados por una epidemia extendida, que se convierte en pandemia especialmente en el sur de Europa. La globalización de los procesos económicos unida a la internacionalización de los procesos de producción del conocimiento son, como bien se encargan de afirmar González, Coca, Valero y Aguilar (2015), los factores más destacados que determinan el porqué de la migración altamente cualificada en la actualidad.

\section{Problemática nacional de la fuga de cerebros en clave internacional}

Tal y como sabemos, desde 2010 la emigración española a distintos países de la Unión Europea y de América, aunque también a destinos más remotos de Asia considerados los nuevos nichos económicos, ha crecido de manera exponencial. Si bien esta es una situación compartida con el resto de países mediterráneos, España destaca por haberlo hecho a un ritmo más acelerado (González-Ferrer, 2013) y, lo que nos resulta aún más interesante, con un nivel educativo de sus migrados superior al de sus vecinos (Ermolieva y Kudeyárova, 2014). Por tanto, ya no estamos frente a un flujo prácticamente exclusivo de trabajadores sin cualificar que busca en la vieja Europa o en la América de las oportunidades un salario por el que subsistir, sino que, por el contrario, existe un considerable capital humano cualificado que persigue una oportunidad laboral que ha sido negada en nuestro país. Es lo que algunos investigadores del Banco Mundial han denominado "la gran recesión" (Izquierdo, Jimeno y Lacuesta, 2014).

Bien es verdad que si atendemos a algunos valores absolutos que nos indican que “(...) en el año 2010 la mayor proporción de migrantes registrado en el régimen general eran profesionales no cualificados: el $21,93 \%$ de personas que trabajan en el ámbito de la hostelería y el 16,54\% que trabajan en el comercio" (González, Coca, Valero y Aguilar, 2015, p.75) y que, por el contrario, aquellos que realizaban actividades científicas e intelectuales suponían tan solo el 5,05\%, nuestra preocupación por una autentica fuga de cerebros no tendría que ser extrema, máxime cuando esta no correlaciona positivamente con la recepción de talentos. Pues bien, tal como se encarga de afirmar Puyol (2014), pese a que el impacto del nuevo "brain drain" es moderado no podemos olvidar las consecuencias que la migración de ese $5 \%$ tiene o, lo que aún más preocupante, puede llegar a tener a nivel social. La débil estructura laboral unida al no menos endeble tejido empresarial inquieta, a la vez que preocupa y obstaculiza, "la transición al modelo económico post-crisis" (Ermolieva y Kudeyárova, 2014, p.1) ya que, como sabemos, hablar de movilidad no significa, únicamente, la colocación de expatriados, sino también la concentración productiva puesto que, como nos indican algunos autores, los empleos se mueven donde las personas con talento se encuentran (Lanvin y Evans, 2015). Se describe así un círculo vicioso que poco contribuye a la salida de la crisis y a la reformulación de políticas de progreso socioeconómico: los cerebros 
expulsados de España "por ventilación”, como nos gusta denominar a esta movilidad, viajan a determinados territorios donde, aun siendo inmigrantes, se les ofrecen grandes oportunidades. Estos países receptores actúan como auténticos "aspiradores sociales" de ese flujo de jóvenes altamente cualificados, previamente esparcidos, no permitiendo, a través de sus adecuadas y satisfactorias políticas sociales y laborales, su retorno. Encontraríamos, en esta situación que resume la tónica general, una excepción que es prácticamente inexistente en el caso que presentamos de España y que sucede cuando la diáspora sigue manteniendo lazos sociales y culturales con su país de origen, gracias a las políticas de apoyo a los emigrados, a la facilidad para la inversión con vistas al retorno y, en otro orden, a la adquisición de conocimientos y experiencia profesional a través de redes de intercambio científico. Esto no deja de ser, cuanto menos, curioso ya que, históricamente, se nos ha reconocido por el arraigo, determinante en nuestra movilidad y nuestro, casi siempre presente, retorno. Tal y como lo describe Nelson (2015) en su disertación sobre los efectos sociales de la fuga de cerebros en España, "the Spanish feel the need to have an even stronger relationship with aging family members. The significance that family holds plays into the decisions professionals make in regards to their careers" (s.p.). Aunque no es el objeto de nuestro trabajo, creemos muy interesante comprobar si, en la actualidad, los valores familiares siempre presentes en nuestra sociedad se han visto modificados por las circunstancias económicas y productivas y el papel que estos tienen en el marco del brain drain. Quizás en otra investigación futura nos adentremos en esta tesis.

Retomando nuestro tópico de trabajo, reconocemos que entre las ideas principales que se extraen del reconocidísimo informe "The Global Talent Competitiveness Index. Talent Attraction and International Mobility 2015-2016", recientemente publicado (Lanvin y Evans, 2015), se encuentra aquella que alude a la influencia que la autonomía fiscal y económica tiene en el desarrollo de estrategias para la atracción y retención de talentos, si bien de manera muy dispar en el territorio español. De su estudio, extraemos que no es comparable, por ejemplo, la capacidad demostrada por la Comunidad Autónoma de Andalucía con la del País Vasco, reseñado como paradigma a nivel mundial -territorio más industrializado de Europa y con el mayor PIB del país-.

En términos generales, observamos cómo España ocupa en el ranking mundial (compuesto por 109 países), que refiere el índice de competencias globales relacionadas con el talento, el puesto 36. Esta posición, a nivel europeo, se traduce en el lugar $24^{\circ}$ (de 37 naciones). Sendas posiciones, mundial y europea, demuestran que nuestro país no está, ni de lejos, en el "top ten" ni tan siquiera cuando analizamos los distintos criterios que componen el índice general; las mejores posiciones internacionales se observan en los índices: crecimiento (Grow, 22), retención (Retain, 29), atracción (Attract, 31) y adquisición de competencias de conocimiento general (GK General Knowledge Skills, 34) y las peores en las competencias laborales y vocacionales (LV Labour and Vocational Skills, 59) y en habilidades (Enable, 43).

No podemos olvidar que, pese a ese conglomerado de elementos que componen el talento, este es cada vez más difícil de describir. Las distintas posibilidades abiertas en el arte y las ciencias, acompañadas de las nuevas maneras de entender la(s) inteligencia(s) de manera plural y múltiple, han propiciado un modelo más flexible de conocimiento, si bien, lo que todas ellas comparten, es la importancia que tiene la movilidad como requisito indispensable para alcanzar esa aptitud basada en la creatividad, la capacidad para adaptarse al medio y para hacer frente a problemas diversos. Siendo así, debemos reconocer que la apertura al exterior, es decir, la recepción de profesionales foráneos que incrementen el prestigio social de la cualificación profesional nacional, independientemente de su origen, es la mejor vía para contrarrestar esa fuga. En este contexto huelga decir que "la movilidad internacional de los trabajadores es posiblemente el motor más potente para la prosperidad mundial y la 
redistribución de los ingresos" (Fanjul, 2014, p.3) y que, por tanto, las políticas nacionales deben cerciorarse de favorecer esas andanzas garantizando, siempre y en todo caso, el retorno. Por el contrario, las políticas en nuestro país procuran, constantemente, llenar las lagunas de formación de sus futuros profesionales - a través de diversas reformas, bien generales (EEES) o bien contextualizadas-, no teniendo en consideración que este esfuerzo debe concentrarse no solo en el proceso de cualificación inicial sino en el de formación en puesto de trabajo, tanto de los trabajadores autóctonos como de los extranjeros. Siendo así, ni conseguimos atraer estudiantes -pese a haber priorizado a nivel político la Educación Superior desatendiendo la Vocacional, seguimos ocupando un más que discreto puesto 47 a nivel mundial- ni profesionales en la medida que sería aconsejable asumiendo que "it is not only whether you open or close the door, it is also whether the house is warm and welcoming" (Lanvin y Evans, 2015, p.104). Siendo así, comprobamos cómo el nivel de desempeño que mide la circulación de cerebros -tanto a nivel recepción como de fuga, de manera independiente- es bastante inferior (la mitad o incluso un tercio) al que obtienen los países más punteros de Europa como son Suiza, Reino Unido, Noruega o Suecia. Ello se traduce en una posición muy baja en el ranking mundial que relega a nuestro país a puestos del último cuartil $\left(74^{\circ}\right.$ en la llegada de cerebros y $83^{\circ}$ en la fuga).

\section{Discusión y conclusiones.}

Tras este panorama general que demuestra por qué España ocupa esas malogradas posiciones internacionales en lo relativo a competencias específicas relacionadas con la movilidad del talento, pasamos a ofrecer algunas medidas que, llevadas a cabo con reconocido éxito en ciertas naciones de nuestro entorno más inmediato, el europeo, estimamos podrían ser tenidas en cuenta para conseguir un verdadero grado de atracción y retención de cerebros internacionales y nacionales, respectivamente.

Tal y como referíamos con anterioridad, la mayoría de países de nuestro continente superan al nuestro en los marcadores del binomio salida-llegada de cerebros. Este tándem es analizado de manera conjunta ya que, como hemos explicado, uno no se entiende sin el otro, considerando que para que se produzca una circulación que sea favorable para el país, la movilidad se debe dar en los dos sentidos, aumentando el prestigio mundial del país en la recepción y, por ende, en el retorno de los migrados nacionales.

En esta línea, comprobamos cómo la estrategia seguida por los que hemos destacado como líderes del ranking, por ser los territorios con mayor capacidad de atracción para los investigadores extranjeros (Domenico y Arenas, 2016), es bien distinta. En lo que se refiere a formación inicial, mientras que los países nórdicos apuestan por una Educación Superior de reconocida calidad en donde la gratuidad forma parte de ese siempre envidiado Estado de Bienestar, Suiza y Reino Unido recurren a un sistema formativo mucho más exclusivista, donde el estatus económico es determinante para su acceso. Estas dos modalidades de ofrecer educación, completamente opuestas en lo que se refiere a su concepción social (en donde podemos aludir a argumentos relacionados con la equidad, con la libertad, con la igualdad...), comparten el reconocimiento a nivel internacional por su alta calidad competencial y por su capacidad para estimular la salida de sus estudiantes al extranjero (Wit, 2002). Entendemos que estas dos últimas circunstancias, además de alguna otra que es, a la vez, causa y efecto de las mismas (por ejemplo, la preferencia y metodología aplicada para el aprendizaje de las lenguas extranjeras), son las que distancian progresivamente y de manera inevitable a nuestro país de los puestos más elevados en esos rankings mundiales. En este sentido, destacamos la situación de Noruega que, con cerca de 6.000 euros per cápita (exactamente 5.824), lidera a nivel mundial la inversión en educación, pero también la de Suiza (3.266), Suecia (3.407), 
Finlandia (2.654) y Reino Unido (1.825), tal y como nos demuestran los "datos macro" ofrecidos por el diario Expansión. Lejos quedan los 1.027 euros per cápita que España invirtió en 2012 suponiendo una caída de cerca del 16\% respecto a su ejercicio anterior. Aunque es verdad que, tal y como informa el Parlamento Europeo (2016), el incremento en gasto público en educación no tiene sentido si no va de la mano de la innovación y el desarrollo, España no hace ni lo uno ni lo otro: reduce su inversión en este apartado a la vez que en aquellos referidos a Ciencia e Investigación.

Esta misma institución supranacional reconoce, al tiempo, que la fuga de cerebros en nuestro continente es preocupante en algunos de sus Estados miembros y que solo a través de políticas integradas, "esto significa más coherencia entre las políticas europeas y nacionales en materia de investigación, y eliminar los obstáculos a la movilidad de los investigadores" (Parlamento Europeo, 2016, p.2), se podrá completar el conocido como Espacio Europeo de Investigación, aún en ciernes. Para este fin se sigue priorizando el papel siempre determinante de la Comisión Europea que “(...) apoyará proyectos para desarrollar nuevos planes de estudios dirigidos a colmar las lagunas de capacidades en innovación" (Parlamento Europeo, 2016, p.3).

La mejora en la capacitación científico-técnica (tanto a nivel universitario como de formación profesional superior), el dominio de, al menos, una lengua extranjera (preferentemente inglés, aunque no solo), la participación en proyectos de investigación y desarrollo internacionales y el ajuste laboral entre la oferta y la demanda son solo algunas de las iniciativas que favorecen ese intercambio cooperativo del que nos habla, con gran acierto y tino, Solanas (2014). De manera más concreta, son ya varios los países de nuestro entorno más inmediato los que han optado bien por la creación de "partenariados" internacionales, tanto de centros de formación de diversa índole como de empresas (Verger, 2013), o bien por la selección exhaustiva de aquellos estudiantes que provienen de fuera de la Unión (imponiéndoles altas tasas y ofreciendo becas competitivas que garanticen la inversión y su permanencia a través de los conocidos "job-search schemes" ingleses), sin olvidar la posibilidad de ofrecer determinados beneficios sociales tanto para aquellos trabajadores que quieren continuar en sus países de origen como para los que, siendo extranjeros, proyectan su futuro profesional en territorio foráneo (Wit y Ripmeester, 2013). Todas estas iniciativas han sido, sin embargo, escasamente reproducidas en nuestro país. Una clara falta de interés por parte de las autoridades y grupos políticos que se han sucedido en el poder proporciona una serie de políticas de "manos atadas" que poco o nada deja hacer a la ciudadanía.

Por lo tanto, estimamos que estas iniciativas internacionales de alto calado y amplia trayectoria deben ser analizadas, interpretadas $y$, en la medida de lo posible, implementadas minuciosamente en el desarrollo de nuestras futuras políticas públicas. Tal y como hemos referido con anterioridad, aunque en los últimos tiempos España se ha preocupado por contener la fuga masiva de sus talentos, el reflejo internacional nos sigue revelando que es preciso encaminar las políticas de formación (y producción) hacia tendencias mucho más abiertas y consecuentes que consigan, por un lado, atraer a los mejores cerebros internacionales para beneficiarse, aunque sea puntualmente, de sus conocimientos y competencias y, por otro, gestar políticas de movilidad internacional que incluyan, como parte de las mismas, el retorno.

Siendo así, nos decantamos por afirmar que el desarrollo de propuestas asentadas en la movilidad (y no en la fuga) garantiza resultados altamente beneficiosos, no solo de manera inmediata, sino también a medio y largo plazo, lo que implica embarcarse en políticas de permanencia, en proyectos de continuidad, en estrategias de previsión. 
Sin embargo, aunque todos los países sufren movimiento de sus cerebros, solo algunos consiguen sacar provecho del mismo. Estas son las naciones del presente, pero esencialmente, las que protagonizarán el futuro. Las políticas de reintegro, el seguimiento y "acompañamiento" de la carrera en el extranjero, la exigencia académica y el valor otorgado a la formación internacional forman parte solo de la conciencia socioeducativa inculcada a los estudiantes y trabajadores de algunos selectos países. El contexto se demuestra, en este punto, esencial para garantizar unos óptimos resultados al igual que lo es el valor que la sociedad otorga a la formación en el extranjero. Desafortunadamente, España no se encuentra todavía entre esos países punteros en movilidad. La tradición migratoria en nuestro país interfiere considerablemente en esa percepción considerando que los que salen lo hacen siempre por falta de posibilidades en el mismo sin barajar, en la mayoría de los casos, que esta movilidad puede resultar una apertura a nuevas metas formativas que deben ser potenciadas y celebradas siempre que incluyan su regreso. Siendo así, estimamos igualmente necesario un cambio en la conciencia social y política que otorgue un mayor valor, sentido y reconocimiento al intercambio de cerebros como parte esencial e innegociable del proceso de construcción social de toda persona. Este debe pasar por un replanteamiento (y posterior dotación) de las políticas, no solo formativas sino también productivas, económicas, fiscales y culturales en el que quede patente el interés, a la vez que la necesidad, de fomentar la movilidad no como fin o como meta, sino como medio para alcanzar una verdadera internacionalización. Únicamente, de este modo, conseguiremos avanzar en la construcción de una auténtica Sociedad Global que nos permita "pensar el presente, rebuscar en el pasado e imaginar el futuro" (Ianni, 1998, p.1).

\section{Referencias bibliográficas}

Alcañíz, M., y Querol, V. (2015). Jóvenes y trayectorias a la vida adulta. Desigualdades, retos y nuevas formas de un contexto en crisis, Recerca. Revista de Pensament i análisi, (16), 7-12, doi: http://dx.doi.org/10.6035/Recerca.2015.16.1

Alcón, E. (2011). La internacionalización de los estudiantes universitarios. La cuestión Universitaria, (7), 32-39. Recuperado de http://calidad.ugr.es/tutoria/materiales_asistentes/lacuestionuniversitaria/!

Brandi, M. C. (2006). La historia del brain drain. Revista Iberoamericana Ciencia, Tecnología y Sociedad, 3 (7), 65-85. Recuperado de http://www.scielo.org.ar/scielo.php?script=sci_arttext\&pid=S185000132006000100005

Castells, M. (1996). La era de la información: economía, sociedad y cultura. Madrid: Editorial Alianza.

Docquier, F., y Rapoport, H. (2012). Globalization, brain drain, and development. Journal of Economic Literature, 50 (3), 681-730, doi: 10.1257/jel.50.3.681

Domenico, M., y Arenas, A. (2016). Researcher incentives: EU cash goes to the sticky and attractive. Nature, 531, 580, doi: 10.1038/531580c

Ermolieva, E., y Kudeyárova, N. (2014). La movilidad internacional de recursos humanos cualificados: nuevas tendencias (el caso de España). Camino Real, 9, 39-55. Recuperado 
http://dspace.uah.es/dspace/bitstream/handle/10017/21938/movilidad_Ermolieva_CR _2014_N9.pdf?sequence=1\&isAllowed=y

Fanjul, G. (2014). VII Informe sobre exclusión social en España 2014. La política migratoria en Europa. Fundación Foessa. Recuperado de http://foessa2014.es/informe/uploaded/documentos_trabajo/04112014003430_8075. pdf

González, A.M., Coca, J., Valero, J.A., y Aguilar, J. (2015). Migración cualificada en España: un análisis empleando una metodología de conjuntos difusos. Lurralde: investigación y espacio, 38, 69-88. Recuperado de http://www.ingeba.org/lurralde/lurranet/lur38/38\%20coca.pdf

González-Ferrer, A. (2013). Zoom político. La nueva emigración española. Lo que sabemos y lo que no. Madrid: Fundación Alternativas.

Ianni, O. (1998). La Sociedad Global. México: Siglo XXI Editores.

Izquierdo, M., Jimeno J.F., y Lacuesta, A. (2014). La emigración de españoles durante la Gran Recesión (2008-2013). Cuadernos económicos de ICE, 87, 223-240. Recuperado de http://www.revistasice.com/CachePDF/CICE_87__6BDA4E47903E58D055F14CA C795721E7.pdf

Lanvin, B., y Evans, P. (ed.) (2015). The Global Talent Competitiveness Index. Talent Attraction and International Mobility 2015-2016. Recuperado de http://globalindices.insead.edu/gtci/documents/INSEAD_2015-16_Full_Book_Ebook.pdf

Luchilo, L. (2006). Movilidad de estudiantes universitarios e internacionalización de la educación superior. Revista CTS, 3 (7), 105-133. Recuperado de http://www.revistacts.net/volumen-3-numero-7/55-dossier/192-movilidad-deestudiantes-universitarios-e-internacionalizacion-de-la-educacion-superior

Nelson, O. (2015). The Social Effects of the Spanish Brain Drain. Social Impact Research Experience (SIRE). Paper 35. Recuperado de http://repository.upenn.edu/sire/35

Parlamento Europeo (2016). Fichas técnicas sobre la unión Europea. La política de innovación. Recuperado de http://www.europarl.europa.eu/ftu/pdf/es/FTU_5.9.7.pdf

Puyol, R. (2014). Expatriados por el empleo: ¿activo o pérdida para España? Nueva Revista de Política, Cultura y Arte, 149, 216-229. Recuperado de http://www.revistasculturales.com/xrevistas/PDF/22/1789.pdf

Reyes, A.E. (2015). Educación y formación en la Unión Europea: análisis del proceso de Bolonia, el Espacio Europeo de Educación Superior, la Estrategia Europa 2020 y el programa ERASMUS+. Derecho y cambio social, 12 (42), pp.1-23. Recuperado de https://dialnet.unirioja.es/descarga/articulo/5456404.pdf

Santos, A. (2013). Fuga de cerebros y crisis en España: los jóvenes en el punto de mira de los discursos empresariales. AREAS. Revista Internacional de Ciencias Sociales, 32, pp.125 - $137 . \quad$ Recuperado de http://revistas.um.es/areas/article/viewFile/192331/158761 
Sassen, S. (2007). A Sociology of Globalization. New York: W.W. Norton.

Sennett, R. (2000). La corrosión del carácter. Barcelona: Anagrama

Solanas, F. (2014). Intercambio cooperativo versus mercantilización competitiva: las políticas de movilidad académica en el MERCOSUR y la Unión Europea. Revista Iberoamericana de Educación Superior (RIES), 5 (12), pp. 3-22, doi: 10.1016/S20072872(14)71940-7

Valero, J.A., Mediavilla, J. J., Valero, I., y Otero, J. (2015). El pasado vuelve a marcar el presente. La emigración española. Papeles de Población, 21 (83), pp.41-74. Recuperado de http://www.redalyc.org/pdf/112/11234130003.pdf

Verger, A. (2013). Políticas de mercado, Estado y universidad: hacia una conceptualización y explicación del fenómeno de la mercantilización de la Educación Superior, Revista de Educación, 360, pp. 268-291, doi: 10-4438/1988-592X-RE-2011-360-111

Wit, H. (2002). Internacionalization of higher education in the United States of America and Europe. A historical, comparative and conceptual analysis. Estados Unidos: Greenwood Publishing Group.

Wit, H. y Ripmeester, N. (2013). Increasing the stay rate of international students. University World News. The global window on higher education, 259. Recuperado de http://www.universityworldnews.com/article.php?story=20130213165216138 